\title{
Prophylactic laser in age-related macular degeneration: the past, the present and the future
}

\author{
Quan Findlay ${ }^{1} \cdot$ Andrew I. Jobling ${ }^{1} \cdot$ Kirstan A. Vessey $^{1} \cdot$ Ursula Greferath $^{1} \cdot$ Joanna A. Phipps ${ }^{1} \cdot$ Robyn H. Guymer $^{2}$. \\ Erica L. Fletcher ${ }^{1}$
}

Received: 18 December 2017 / Accepted: 14 January 2018 / Published online: 9 March 2018

(c) The Royal College of Ophthalmologists 2018

\begin{abstract}
The presence of drusen in the posterior eye is a hallmark feature of the early stages of age-related macular degeneration and their size is an indicator of risk of progression to vision-threatening forms of the disease. Since the initial observations that laser treatment can resolve drusen, there has been great interest in whether laser treatment can be used to reduce the progression of age-related macular degeneration. In this article, we review the development of lasers for the treatment of those with age-related macular degeneration. We provide an overview of the clinical trial results that demonstrated drusen resolution but that had mixed effects on progression of disease. In addition, we provide a summary of the recent developments in pulsed lasers that are designed to reduce the energy applied to the posterior eye to provide the therapeutic effects of conventional continuous wave lasers while reducing the secondary tissue effects.
\end{abstract}

\section{Introduction}

Age-related macular degeneration (AMD) is the predominant cause of irreversible visual loss in people over 55 years of age, accounting for approximately $50 \%$ of legal blindness in most developed countries including the United States, Europe and Australia [1,2]. There are two forms of advanced AMD, which are characterised by the development of either choroidal neovascularisation (CNV), the 'wet' exudative form, or geographic atrophy (GA), the 'dry' atrophic form. Regardless of the type of advanced AMD, there are common features that occur in the early stages of the disease. Notably, extracellular deposition of lipid-rich deposits called drusen accumulate between the retinal pigment epithelium (RPE) and Bruch's membrane, an elastinrich layer separating the RPE from the choroid [3, 4]. The presence of drusen is a hallmark feature of the early stages of AMD and their type, size and number influence the risk

Robyn H. Guymer

rh.guymer@unimelb.edu.au

1 Department of Anatomy and Neuroscience, The University of Melbourne, Parkville 3010 VIC, Australia

2 Centre for Eye Research Australia, University of Melbourne Royal Victorian Eye and Ear Hospital, Parkville VIC, Australia of progression of AMD [5]. Drusen greater than $125 \mu \mathrm{m}$ in size are often referred to as soft or confluent drusen. These are associated with the highest risk of progression, compared to smaller sized drusen, particularly when present in both eyes [5]. The period of time between when drusen are observed in the posterior eye and progression to visionthreatening forms of the disease occur can be long, often occurring over decades, thus providing considerable opportunities for intervention.

Although the precise mechanism(s) that lead to the formation of drusen are not completely known, anomalies in the transport of lipid through the RPE in combination with changes in structure and thickness of Bruch's membrane are thought to be important. Indeed, there is gradual accumulation of lipoprotein within Bruch's membrane that is associated with increased thickness and a reduced capacity for nutrient and waste transport across the RPE [6]. Thickening of Bruch's membrane is thought to be a critical step in the anomalies in visual sensitivity that occur from an early stage of AMD [7], and may also be important in the subsequent inflammatory and/or angiogenic anomalies that develop as part of progression to advanced AMD. Thus, strategies for reducing drusen, which may be merely a biomarker for positive changes in Bruch's membrane, and/ or those which have positive effects on Bruch's membrane thickness, continue to be evaluated for their potential in reducing progression of AMD. 
To date, despite attempts, there is little to offer in the management strategies for AMD that specifically prevent progression of AMD from the early stages to the late visionthreatening complications of wet or dry AMD. Apart from ceasing to smoke and maintaining a healthy lifestyle and diet [8], various supplements have been investigated. The Age-Related Eye Disease Study (AREDS) showed some benefit of anti-oxidant vitamin supplementation in some early-stage AMD patients to slow progression to advance AMD [9]. However, for many patients, anti-oxidant vitamin supplementation showed no beneficial effect. The later AREDS 2 study modified the original AREDS formulation to include lutein, zeaxanthin and omega-3 fatty acids, but there was no further benefit on preventing the development to advanced AMD [10].

Over the last 20 years, there has been increasing interest in the potential for ophthalmic laser treatment to reduce drusen number and size, thereby reducing progression. The aim of this article is to summarise the development of ophthalmic lasers in the management of the early stages of AMD. We begin with a historical perspective outlining the initial observations that laser application to the posterior eye was associated with drusen resolution. We then consider the results of the randomised clinical trials evaluating the prophylactic use of continuous wave lasers for reducing progression of AMD. Finally, we summarise the recent development of subthreshold lasers and the potential these lasers have for managing those with early AMD.

\section{The origin of laser photocoagulation as a potential prophylactic treatment for AMD}

Drusen resolution following laser application to the macular area was first observed by Gass in 1973 [11]. He noted that following laser photocoagulation of eyes with $\mathrm{CNV}$ or pigment epithelium detachment using a ruby or argon laser, large drusen in adjacent areas resolved, and that the appearance of the macula was restored to almost normal appearance in a manner that did not affect visual acuity. These incidental findings heralded the notion that laser treatment of retinae could lead to resolution of drusen and potentially reduce progression to advanced forms of AMD.

The initial studies examining the effect of continuous wave laser treatment on drusen resolution and progression of AMD were largely conducted in small groups of patients with limited statistical power. Laser burns were applied to the retina either directly to the drusen, or in a predefined pattern across the macula and the laser energy was calibrated to cause a minimal visible reaction. Cleasby et al. [12] applied photocoagulation directly to drusen in one eye using an argon green laser in patients with bilateral drusen $(N=25)$ and patients with exudative AMD $(N=29)$. Drusen reduction was seen in most treated eyes during a follow- up period of 11-65 months. However, there was no difference in the rate of $\mathrm{CNV}$ development after laser treatment compared to non-lasered eyes in patients with bilateral drusen. Wetzig [13] and colleagues conducted a retrospective clinical study and treated patients with large drusen and recent visual changes including metamorphopsia or decreased visual acuity. They showed that treatment with scatter-pattern photocoagulation, which neither targeted nor avoided drusen, resulted in drusen reduction. Importantly, this was the first study that showed a protective benefit to visual acuity, with the stabilisation of vision in $52 \%$ of 42 lasered eyes. Overall, these studies demonstrated that application of continuous wave argon laser that induced a minimal visible reaction lead to resorption of drusen in a majority of eyes. What was not clear was the mechanism by which drusen resolution occurred and whether the laser treatment led to reduced progression of disease.

\section{Histopathological changes induced by thermal laser application in eyes with drusen}

In concert with the clinical observations that laser application could resolve drusen and potentially reduce progression of disease were a series of studies important for gaining a better understanding of the cellular effects induced by the laser-induced treatment. Laser application to the posterior eye had effects on the RPE, infiltrating macrophages, the structure of the choriocapillaris and Bruch's membrane.

Laser energy is absorbed by pigments in the RPE, predominantly melanin, leading to the death of RPE cells as well as the spreading of thermal effects to surround tissues. The spread of heat anteriorly can cause injury to the inner retina and the nerve fibre layer, while the spread of heat posteriorly can cause breaks in Bruch's membrane. However, in addition to the death of dysfunctional RPE cells, Frennesson and Nilsson [14] suggested that laser photocoagulation, at low power, could beneficially lead to the proliferation of newer and more functionally active RPE cells which could better phagocytose drusen material.

In addition to changes in the RPE, treatment of the posterior eye with a continuous wave laser also induces changes on Bruch's membrane and infiltrating macrophages. Sigelman [15] suggested that laser photocoagulation increases the permeability of Bruch's membrane so as to improve the egress of drusenoid debris. Bruch's membrane thickens with age in response to an accumulation of lipid, among other age-related changes. This in turn presents a hydrophobic barrier that inhibits the flow of waste products from the RPE to the choroid, as well as nutrients from the choroid to the RPE [16]. Laser application may cause contraction of collagen and elastin within Bruch's membrane disrupting the hydrophobic properties of this barrier, thereby enhancing transport conductivity [17]. In addition, 
Table 1 Summary of major randomised clinical trials evaluating laser prophylaxis for management of AMD

\begin{tabular}{lllll}
\hline Major RCT & $\begin{array}{l}\text { Year of enrolment } \\
\text { commencement }\end{array}$ & Number of participants & Location & References \\
\hline CAPT & 1999 & 1052 total & US & CAPT Research Group, 2006 [25] \\
CNVPT & 1994 & $\begin{array}{l}156 \text { bilateral, 120 } \\
\text { unilateral }\end{array}$ & US & CNPT Research Group, 1998, 2003 \\
[21, 22] & Owens et al., 2003, 2006 [23, 24] \\
DLS & 1996 & $\begin{array}{l}105 \text { bilateral, 177 } \\
\text { unilateral }\end{array}$ & UK, Germany, Australia & Ow total \\
Frennesson 2009 & 1998 & $\begin{array}{l}\text { 639 bilateral, 244 } \\
\text { unilateral }\end{array}$ & Scandinavia & Fs \\
PTAMD & 1996 & & & Friberg et al., 2006, 2009 [26, 27] \\
\hline
\end{tabular}

Guymer et al. [18] showed that laser photocoagulation led to an increase in protrusions of choroidal endothelial processes into Bruch's membrane, which they proposed increased the vascular surface area, which could aid in the removal of drusenoid material. Duvall and Tso [19] examined the ultrastructural changes associated with drusen resolution in a rhesus monkey following laser treatment. They observed infiltration phagocytic cells, especially macrophages into the lasered and the neighbouring areas. Notably, macrophages containing phagosomes filled with drusenoid material and photoreceptor debris were found clustered around the RPE and damaged photoreceptors within the subretinal space. The authors attributed the removal of drusen material to the increased phagocytic activity of macrophages.

\section{Randomised controlled trials evaluating prophylactic thermal laser therapy to slow progression in AMD}

Prophylatic laser treatment has been investigated in groups of patients with large bilateral drusen $(>125 \mu \mathrm{m}$ in size) or those with $\mathrm{CNV}$ in one eye and large drusen in the fellow eye because these two groups of patients represent those at greatest risk of vision loss from disease progression. The first randomised controlled trial (RCT) was reported by Figueroa et al. [20] in 1994. They prospectively recruited 46 patients with confluent soft drusen in either one (unilateral group) or both (bilateral group) eyes and directly targeted drusen in the temporal macula with an argon green continuous wave laser. Patients in the bilateral group (30 patients) had one eye treated, with the second eye serving as a non-lasered control. Patients in the unilateral group (16 patients) had CNV in one eye, and only the contralateral eye, with soft drusen, was treated. Temporal drusen which were directly treated by laser resolved over a mean period of 3.5 months, but untreated drusen in subfoveal and nasal macula also resolved over a mean period of 8.5 months. After a mean of 3-year follow-up, CNV did not occur in the treated eyes, but was seen in one control eye in the bilateral group. In the unilateral group, CNV occurred in $3(18 \%)$ of treated patients. The investigators concluded that prophylactic laser treatment may have a role in reducing the risk of progression in patients with high-risk bilateral soft drusen and that a larger study was required to allow more definitive conclusions to be made.

Frennesson and Nilsson [14] performed a prospective randomised controlled study of 38 patients using an argon laser (100-200 $\mathrm{mW}$ for $0.05 \mathrm{~s}$ duration) on and between drusen in a temporal horseshoe-shaped pattern $(>500 \mu \mathrm{m}$ outside the foveal centre). Nineteen eyes were allocated to each experimental group (treatment: 13 with bilateral drusen and 6 fellow eyes; control: 9 with bilateral drusen, 7 fellow eyes and 3 contralateral eyes with optic neuropathy or post encephalitic maculopathy). Drusen reduction was significantly reduced in eyes that had laser treatment in contrast to an increase in drusen area in control eyes $(P<0.01)$. At the 8-year follow-up time point, $\mathrm{CNV}$ was observed in 9 of the 16 control eyes ( 4 bilateral drusen eyes and 5 fellow eyes) compared with 2 of the 13 treated eyes (both bilateral drusen eyes). Atrophy was seen in one eye in the control group. This study indicated that the prophylactic application of laser to soft drusen may be beneficial in reducing the development of $\mathrm{CNV}$, although with only a small sample size, definitive conclusions could not be reached.

These initial small-scale RCTs reported drusen reduction following argon green laser treatment. More importantly, they demonstrated some clinical benefit and highlighted the potential for preventing the progression to advanced AMD. Despite the limitation of small cohort sizes, which made the reported positive results difficult to interpret, these smallscale RCTs provided the impetus to proceed to larger multicentre RCTs.

\section{Large RCT involving thermal laser at subthreshold intensity}

A number of large multicentre randomised clinical trials have been conducted to assess whether prophylactic laser 
treatment reduced progression of AMD in those at highest risk (summarised in Table 1). In addition, in some studies effect of prophylactic laser treatment on visual function (e.g, visual acuity, contrast sensitivity, reading ability) was also considered. The Choroidal Neovascularization Prevention Trial (CNVPT) Research Group [21, 22] conducted two studies (Bilateral Drusen Study and Fellow Eye Study) in the United States. A total of 120 patients with exudative AMD in one eye and more than 10 drusen $(>63 \mu \mathrm{m})$ in the fellow eye were enrolled in the Fellow Eye Study; 156 patients without exudative AMD and with more than 10 drusen $(>63 \mu \mathrm{m})$ in both eyes were enrolled in the Bilateral Drusen Study. In the Bilateral Drusen Study, patients had one eye randomised to laser treatment, with the second eye acting as a control. In the Fellow Eye Study, the eye not affected by exudative AMD was randomised to either laser treatment (59 patients) or control (61 patients). In both studies the treatment groups received 20 laser spots more than $750 \mu \mathrm{m}$ from the foveal centre using an argon laser with the power titrated to a light grey-white intensity. Consistent with previous studies, significant drusen reduction was seen following laser photocoagulation. Furthermore, associated improvements in visual acuity and contrast sensitivity were also seen in the treated eyes at 12 months. However, at 12-18 months, patients in the Fellow Eye Study who had received laser had a significantly higher incidence of CNV (10 of 69 treated eyes) compared to observation ( 2 of 61 control eyes). It was noted that eyes which had received laser photocoagulation at higher intensity were more likely to develop CNV. However, over a longer term ( $>30$ months), the incidence of both CNV and geographic atrophy was the same across the two experimental groups. Despite no difference between the long-term incidence of advanced AMD in both groups, the studies were halted due to safety concerns for increased $\mathrm{CNV}$ incidence in the short term.

Laser-induced CNV was also observed in the Drusen Laser Study [23, 24]. In all, 282 patients were recruited into two groups: 177 in the unilateral arm (soft drusen in one eye and exudative AMD in the second eye) and 105 in the bilateral arm (soft drusen in both eyes). Prophylactic laser photocoagulation with an argon green/yellow dye laser $(0.2 \mathrm{~s}, 65-120 \mathrm{~mW})$ was performed in two rings (12 laser spots in total) $750 \mu \mathrm{m}$ and $1500 \mu \mathrm{m}$ from the centre of the fovea. In the unilateral group, $29.7 \%$ (27 of 91) of treated patients developed CNV compared with $17.65 \%$ (15 of 85 ) of the observed patients after 3 years $(P=0.061)$ with 6 months earlier onset of $\mathrm{CNV}$ seen in patients who received laser treatment $(P=0.05)[23,24]$. In the bilateral group, the incidence of $\mathrm{CNV}$ was also higher in the lasertreated $(11.6 \%, 12$ of 103) compared to the fellow eyes (6.8\%, 7 of 103), but again this was not significant $(P=0.225)$ and there was no difference in $\mathrm{CNV}$ onset.
While the differences in CNV incidence were not statistically significant, the investigators also suspended patient recruitment due to concerns for laser-induced $\mathrm{CNV}$.

The largest randomised controlled trial to date, the Complications of Age-Related Macular Degeneration Prevention Trial (CAPT) [25], began following findings of the CNVPT to specifically investigate laser-induced complications associated with prophylactic laser treatment in patients with AMD. A total of 1052 patients with bilateral drusen were enrolled into the study, with one eye selected randomly for laser treatment (argon green $512 \mathrm{~nm}, 60$ spots in a grid pattern between 1500 and $2500 \mu \mathrm{m}$ from the foveal centre, $0.1 \mathrm{~s}$ ) with the fellow eye allocated for observation. Given that in the CNVPT, CNV incidence was higher in eyes receiving more intense laser burns, the CAPT trial was conducted with low-intensity laser burns that were barely visible. After 5-6 years of follow-up, the investigators concluded that low-intensity laser application did not have any significant effect on visual acuity nor did it change the risk of developing either GA or CNV. Drusen reduction $(\geq 50 \%)$ was seen in $41.8 \%$ of treated eyes compared with $31.2 \%$ in observation eyes at 5 years. The cumulative incidence for choroidal neovascularization was $13.3 \%$ for both groups and the 5-year cumulative incidence for GA was $7.4 \%$ and $7.8 \%$ for control and treated eyes, respectively.

The Prophylactic Treatment of Age-Related Macular Degeneration (PTAMD) $[26,27]$ trial was initiated following results from the smaller trial performed by Olk et al. [28], the first group to use the infrared diode laser. This study recruited 244 patients to the unilateral arm (CNV in one eye and $>5$ drusen in the fellow eye) and 639 patients to the bilateral arm ( $>5$ drusen in each eye). Patients in each arm were randomised to a treatment and control groups. A low-intensity infrared $(810 \mathrm{~nm}, 0.1 \mathrm{~s})$ diode laser was used to create a grid of 48 spots in 4 concentric circles 750 to $2240 \mu \mathrm{m}$ from the foveal centre in the treatment group. Substantial drusen reduction was seen in both study arms $(P<0.001)$, but no visual acuity benefit was gained in the long term ( $>3$ years). In the unilateral arm, the cumulative rate of $\mathrm{CNV}$ at 18 months was significantly higher $(P=0.05)$ in the treated group (19.7\%) compared to control (4.9\%). In the bilateral arm, $\mathrm{CNV}$ rate did not differ between treated and untreated eyes at 3 years. The incidence of GA was not assessed in this trial. The investigators concluded that prophylactic thermal laser treatment of drusen did not prevent the development of CNV, but could even increase the risk of development of $\mathrm{CNV}$ in patients who are already affected by exudative AMD in the contralateral eye.

Frennesson and the Prophylactic Laser Treatment Study Group [29] conducted a multicentre Nordic study involving 135 patients with a follow-up period of 4 years. A total of 
67 patients were randomised to a treatment group (argon green, $0.04 \mathrm{~s}$ ) and 68 patients to the control group. Similar to previous studies, patients were further assigned to two arms (bilateral and unilateral) based on the number of eyes affected by soft drusen. At 4 years, there was no statistical difference $(P=0.2-0.3)$ in $\mathrm{CNV}$ rate between the treated (4 from 55 eyes in the bilateral group, 3 from 13 eyes in the unilateral group) and the control group ( 3 from 54 eyes in the bilateral group and 2 from 14 eyes in the unilateral group). Visual acuity was reduced from baseline in all groups but there were no significant differences between the treated and control groups. Similar to the CAPT and PTAMD trials, the authors concluded that mild prophylactic laser treatment of soft drusen had neither beneficial nor harmful effects on those with AMD.

Despite the many trials conducted to investigate the effect of prophylactic laser treatment for AMD using conventional thermal laser therapy, the results have been inconclusive, with several studies $[14,17,28]$ demonstrating clinical benefits whereas others $[22,30]$ showed acceleration of $\mathrm{CNV}$ progression. Recently, two systematic reviews of the randomised clinical trials have been conducted to assess the potential for argon laser treatment to reduce the progression of AMD [31, 32]. These two metaanalyses that include over 2000 patients in 11 randomised clinical trials showed that although laser treatment reduced drusen (odds ratio $=9.16$ ) [32], there was no evidence for slowing or accelerating the progression of AMD [32].

In the majority of the multicentre RCTs, the main recurring concern was the perceived increased risk of laserinduced CNV. Indeed, because of the reported risk of CNV at 12-18 months in the CNVPT trial, the published results later led to the cessation of other major RCTs investigating lasers for their potential to reduce the progression of AMD. From various clinical and histopathological studies, it is now understood that inherent in the thermal laser response is an inflammatory reaction precipitated by thermal damage which results in coagulative necrosis of the targeted and neighbouring retinal tissue $[33,34]$. It is possible that this inflammatory response may increase the risk of $\mathrm{CNV}$ development. As such, the need for laser intervention that delivers the beneficial effect, without the accompanying inflammatory side effect, has led to the development of subthreshold lasers.

\section{The development of subthreshold laser treatments}

Laser parameters such as wavelength, power, spot size and pulse duration can be altered to reduce the amount of thermal energy delivered so as to limit tissue damage. These modifications have led to recent developments in subthreshold laser treatment that include using subthreshold diode micropulse lasers, selective retinal therapy using micropulse lasers and nanosecond lasers. Broadly, these lasers may be able to achieve the therapeutic benefits of thermal lasers while minimising retinal damage by delivering light energy as very short pulses [35].

Selective retinal therapy (SRT) involves applying a train of laser pulses that are in the microsecond range [35]. Application of short laser pulses to the posterior eye induces a high temperature within RPE cells that is thought to cause microbubble formation around melanosomes that leads to the selective loss of RPE cells [36]. Importantly, the background temperature increase remains at sublethal levels reducing collateral damage to neighbouring tissues. Cellular effects of SRT have been evaluated in a number of laboratory studies confirming the selectivity of RPE cell loss and the limited effect on the neighbouring neural retina $[37,38]$. In addition, retinal function in humans has been evaluated over a 1-year period using microperimetry and showed no anomalies in regions immediately above the lasered regions over a 1-year period [39]. The only clinical application of SRT reported to date has been a small nonrandomised study in patient with diabetic macula oedema [40].

\section{Subthreshold diode laser treatment}

The subthreshold diode micropulse laser (SDM) delivers energy via multiple repetitive short pulses, which is released in bursts lasting 100-500 ms. Importantly, the time between laser bursts (referred to as the duty cycle) is long enough to prevent thermal energy being dissipated into neighbouring tissue. In addition, a longer wavelength near-infrared laser (i.e., $810 \mathrm{~nm}$ diode laser) further minimises thermal diffusion into tissues. Clinically, the injury profile of the subthreshold diode micropulse laser is not well established, although it has been reported that there are limited discernible effects on the retina $[41,42]$ when viewed with standard ophthalmological imaging techniques (e.g., optical coherence tomography, fluorescein angiography). A few retrospective studies, utilising the micropulse laser, have been conducted on patients with AMD. Luttrull and Margolis [43] conducted a non-randomised study without control subjects on 108 patients with AMD using a panmacular micropulse laser (1800-3000 spots). At 1 month after laser application, they reported electrophysiological enhancement of inner retina and ganglion cell function using pattern electroretinography [43]. A few other investigators have also reported clinical benefits and explored different indications for the micropulse laser in various small-scale studies $[44,45]$. However, in all of these studies, the focus was on derived benefits in advanced AMD rather than the prophylactic treatment in early AMD. The capability of the SDM laser to arrest or slow progression of AMD has not yet been explored in any large-scale randomised clinical study. 

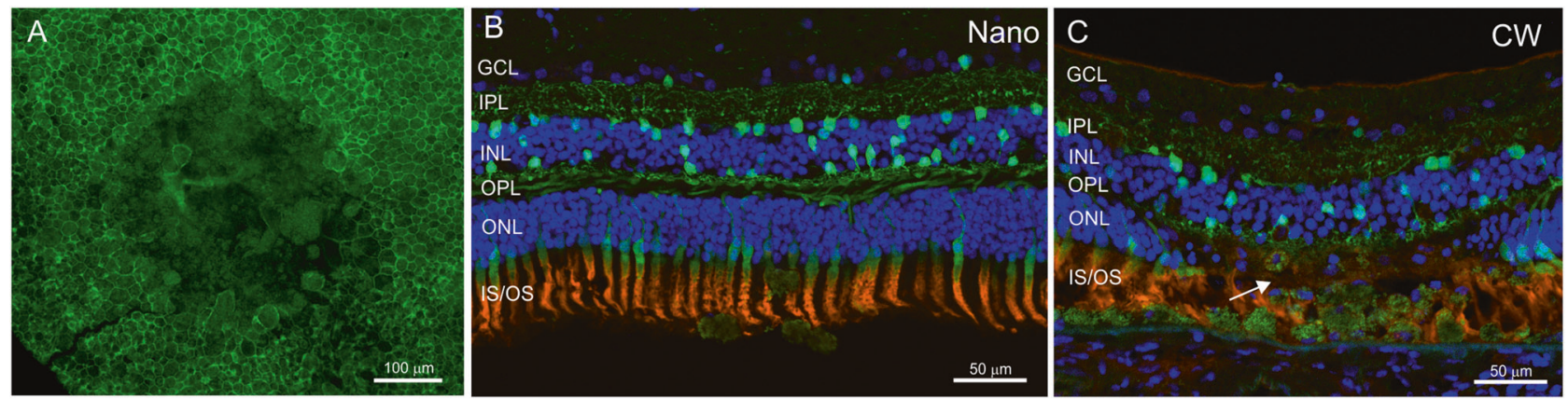

Fig. 1 Effect of laser treatment on the structure of the human RPE and retina. a Flatmounted region of RPE that had received nanosecond laser treatment 5 days prior to exenteration and is labelled with the F-actin marker, phalloidin. The nanosecond laser induces selective ablation of a small area of RPE. b Vertical section of human retina in the region that had received nanosecond laser treatment 5 days prior. Cones are immunolabeled with peanut agglutinin (red), other retina neurons are labelled with calbinin (green) and all somata are labelled with the nuclei stain, bisbenzamide (Hoechst). There is no visible

\section{Development of nanosecond laser treatment for prophylactic treatment in AMD}

More recently, a nanosecond laser has been developed, which delivers energy in 3-nanosecond pulses through a fine speckled-pattern distribution laser beam [46]. Clinically, the nanosecond laser has been employed in a pilot study involving 50 patients with bilateral drusen $>125 \mu \mathrm{m}$ (intermediate AMD) [47]. Treatment involving application of 12 laser spots within the macula of one eye was associated with a reduction in drusen in $44 \%$ of treated eyes and $22 \%$ in the fellow eye over a 12-month period [47, 48]. Macular sensitivity measured by flicker perimetry also improved by a mean of $9 \mathrm{~dB}$ at 3 months post laser. When compared to a historical control group of 58 patients with intermediate AMD, at the 24 -month time point, $35 \%$ of the treated eyes had drusen regression compared with $11 \%$ of eyes in the natural history cohort $(P<0.01)$ [40]. Drusen resolution is known to precede the development of RPE atrophy, a hallmark of disease progression [49, 50]. In order to ascertain whether drusen resolution induced by nanosecond laser treatment occurred because of disease progression, rather than the laser treatment, changes in fundus autofluorescence were evaluated. Fundus autofluorescence was quantified in the areas of the macula where drusen resolved and compared to adjacent regions where drusen were stable. In the majority $(75 \%)$ of patients, fundus autofluorescence images showed no changes in intensity, suggesting that there were no anomalies induced in underlying RPE (e.g., such as atrophy) following laser treatment suggestive of progression of disease [48].

It has been hypothesised that the nanosecond laser achieves photostimulation of the RPE that triggers a woundhealing response in adjacent RPE cells. The nanosecond destruction of any of the retinal layers in the laser treated region. c Vertical section of the human retina in the region that had received continuous wave laser treatment 5 days prior. Cones are labelled with peanut agglutinin (red), and other retinal neurons with calbinin (green). All nuclei are stained with bisbenzamide (Hoechst; blue). The outer nuclear layer is absent in the area that received laser treatment (white arrow). GCL ganglion cell layer, IPL inner plexiform layer, INL inner nuclear layer, OPL outer plexiform layer, ONL outer nuclear layer, IS/ OS inner segments/outer segments

laser induces discrete and selective RPE cell death (see Fig. 1a) $[46,48]$ that is thought to be through a mechanism involving the production of microbubbles around melanosomes $[41,51,52]$. The injury profile of the nanosecond pulsed laser on RPE in animal models, in human explants and in human eyes treated before exenteration of the eye have been reported [46, 48, 51, 52]. These studies have shown that even at high suprathreshold energy levels, nanosecond laser treatment causes significantly less collateral damage to neighbouring tissue when compared with the conventional thermal lasers [48]. When applied at clinical therapeutic energy levels $\left(109 \mathrm{~mJ} / \mathrm{cm}^{2}\right.$ per pulse), damage is confined exclusively to the RPE, with preservation of the overlying neuroretina. This is observed in human retinae using optical coherence tomography as well as in histological analysis of human and mouse retinae. Figure $1 \mathrm{~b}, \mathrm{c}$ show the structure of the human retina 5 days after a nanosecond or continuous wave laser treatment, respectively. Retinal structure is preserved following application of a nanosecond laser burn, whereas following a continuous wave laser spot, the outer nuclear layer is almost entirely absent.

Thickening of Bruch's membrane is thought to be central to the progression of AMD. It is a dynamic structure that is constantly formed and degraded. Degradation is facilitated by matrix metalloproteinases (MMPs), enzymes secreted by the RPE that breakdown collagen and other constituents of the extracellular matrix. There is some evidence that nanosecond laser has the potential to ameliorate the pathological thickening of Bruch's membrane [48]. Zhang et al. [53] showed an increase in the level of MMP-9 and MMP-2 secreted by cultured human RPE following application of the nanosecond laser. Furthermore, treatment of 9-month-old ApoEnull mice with the nanosecond laser 
prevented thickening of Bruch's membrane over a 4-month period compared to non-lasered ApoEnull and control (C57B16) mice. Moreover, the laser-induced changes in Bruch's membrane thickness were associated with changes in the expression of a range of genes associated with the extracellular matrix including MMP-2 and MMP-3 [48]. These studies indicate that the nanosecond laser induces selective ablation of the RPE without inducing secondary effects on the overlying or neighbouring neural retina. Furthermore, these studies also suggest that the nanosecond laser may have other undiscovered positive effects on the surviving and/or newly generated RPE cells which may contribute to debris clearance and/or thinning of Bruch's membrane.

Overall, the results to date investigating the effect on the retina of the nanosecond laser show that application of this laser to patients with intermediate AMD is associated with a reduction in drusen in both the treated eye and in some cases fellow eyes. In addition, nanosecond laser improves macular sensitivity. In a mouse model nanosecond laser treatment reduces Bruch's membrane thickness via alterations in ECM gene expression. Importantly, it was able to achieve these effects while preserving retinal structure and without causing an overt inflammatory immune response or hastening the progress of retinal atrophy or CNV. The longterm effect of nanosecond laser treatment in preventing progression to advanced AMD remains to be determined and is currently under investigation in a large multicentre, randomised clinical trial [54].

\section{Conclusion}

After decades of fundamental research and clinical trials, the prophylactic use of laser therapy to prevent or reduce progression of AMD remains under investigation. However, significant progress has been made in the field of laser technology and modalities, which has created new opportunities and has defined potential new roles for the use of modern lasers in AMD. From the major RCT, it is now well established that photocoagulation using the traditional thermal laser results in clearance of drusen, but does not alter the risk of progression to advanced AMD. The interest now is in the short pulse duration lasers, delivered in a subthreshold manner in the attempt to garner the beneficial effects of the laser without any damaging inflammatory effect. These novel lasers aim to produce photostimulation which selectively targets the RPE without the accompanying collateral retinal damage. As such, these new modalities in laser technology may provide promising treatments of AMD through RPE-mediated mechanisms which had remained unexplored initially with thermal lasers. Currently, the Laser Intervention in Early Age-Related Macular
Degeneration Study (LEAD), a multi-centre randomised clinical trial using a nanosecond laser to investigate progression of AMD in 240 participants, is underway. We are unaware of other large randomised clinical trials using other subthrehold lasers, although smaller cohort studies and treatments continue. The clinical benefit of prophylactic laser application to treat AMD, and which sub-groups of AMD should be treated, will be further clarified once the results from the LEAD trial and other upcoming studies emerge in the near future.

Acknowledgements We would like to thank Lidia Trogrlic for assistance in preparing Fig. 1. The work described in this review was supported by grants from the National Health and Medical Research Council (Australia) and the Australian Research Council.

\section{Compliance with ethical standards}

Conflict of interest The authors declare that they have no conflict of interest.

\section{References}

1. Lim LS, Mitchell P, Seddon JM, Holz FG, Wong TY. Age-related macular degeneration. Lancet. 2012;379:1728-38.

2. Mitchell P, Smith W, Attebo K, Wang JJ. Prevalence of agerelated maculopathy in Australia. The Blue Mountains Eye Study. Ophthalmology. 1995;102:1450-60.

3. Booij JC, Baas DC, Beisekeeva J, Gorgels TG, Bergen AA. The dynamic nature of Bruch's membrane. Prog Retin Eye Res. 2010;29:1-18.

4. Pikuleva IA, Curcio CA. Cholesterol in the retina: the best is yet to come. Prog Retin Eye Res. 2014;41:64-89.

5. Ferris FL 3rd, Wilkinson CP, Bird A, Chakravarthy U, Chew E, Csaky $\mathrm{K}$, et al. Clinical classification of age-related macular degeneration. Ophthalmology. 2013;120:844-51.

6. Guymer R, Luthert P, Bird A. Changes in Bruch's membrane and related structures with age. Prog Retin Eye Res. 1999;18:59-90.

7. Fraser RG, Tan R, Ayton LN, Caruso E, Guymer RH, Luu CD. Assessment of retinotopic rod photoreceptor function using a dark-adapted chromatic perimeter in intermediate agerelated macular degeneration. Invest Ophthalmol Vis Sci. 2016;57:5436-42.

8. Bhutto I, Lutty G. Understanding age-related macular degeneration (AMD): relationships between the photoreceptor/retinal pigment epithelium/Bruch's membrane/choriocapillaris complex. Mol Asp Med. 2012;33:295-317.

9. Age-Related Eye Disease Study Research Group A randomized, placebo-controlled, clinical trial of high-dose supplementation with vitamins $\mathrm{C}$ and $\mathrm{E}$, beta carotene, and zinc for age-related macular degeneration and vision loss: AREDS report no. 8. Arch Ophthalmol. 2001;119:1417-36.

10. Age-Related Eye Disease Study 2 Research Group. Lutein +zeaxanthin and omega-3 fatty acids for age-related macular degeneration: the Age-Related Eye Disease Study 2 (AREDS2) randomized clinical trial. JAMA. 2013;309:2005-15.

11. Gass JD. Drusen and disciform macular detachment and degeneration. Arch Ophthalmol. 1973;90:206-17.

12. Cleasby GW, Nakanishi AS, Norris JL. Prophylactic photocoagulation of the fellow eye in exudative senile maculopathy. A preliminary report. Mod Probl Ophthalmol. 1979;20:141-7. 
13. Wetzig PC. Treatment of drusen-related aging macular degeneration by photocoagulation. Trans Am Ophthalmol Soc. 1988;86:276-90.

14. Frennesson C, Nilsson SE. Prophylactic laser treatment in early age related maculopathy reduced the incidence of exudative complications. Br J Ophthalmol. 1998;82:1169-74.

15. Sigelman J. Foveal drusen resorption one year after perifoveal laser photocoagulation. Ophthalmology. 1991;98:1379-83.

16. Grindle CF, Marshall J. Ageing changes in Bruch's membrane and their functional implications. Trans Ophthalmol Soc UK. 1978;98:172-5. 1978

17. Little HL, Showman JM, Brown BW. A pilot randomized controlled study on the effect of laser photocoagulation of confluent soft macular drusen. Ophthalmology. 1997;104:623-31.

18. Guymer RH, Hageman GS, Bird AC. Influence of laser photocoagulation on choroidal capillary cytoarchitecture. Br J Ophthalmol. 2001;85:40-46.

19. Duvall J, Tso MO. Cellular mechanisms of resolution of drusen after laser coagulation. An experimental study. Arch Ophthalmol. 1985;103:694-703.

20. Figueroa MS, Regueras A, Bertrand J. Laser photocoagulation to treat macular soft drusen in age-related macular degeneration. Retina. 1994;14:391-6.

21. Sternberg P, Aaberg TM, Martin D, Saperstein D, Hyatt M, Gilman $\mathrm{J}$, et al. Laser treatment in fellow eyes with large drusen: updated findings from a pilot randomized clinical trial. Ophthalmology. 2003;110:971-8.

22. Choroidal Neovascularization Prevention Trial Research Group. Laser treatment in eyes with large drusen. Short-term effects seen in a pilot randomized clinical trial. Choroidal Neovascularization Prevention Trial Research Group. Ophthalmology. 1998;105:11-23.

23. Owens SL, Bunce C, Brannon AJ, Wormald R, Bird AC, Drusen Laser Study Group Prophylactic laser treatment appears to promote choroidal neovascularisation in high-risk ARM: results of an interim analysis. Eye (Lond). 2003;17:623-7.

24. Owens SL, Bunce C, Brannon AJ, Xing W, Chisholm IH, Gross $\mathrm{M}$, et al. Prophylactic laser treatment hastens choroidal neovascularization in unilateral age-related maculopathy: final results of the drusen laser study. Am J Ophthalmol. 2006;141:276-81.

25. Complications of Age-Related Macular Degeneration Prevention Trial Research Group. Laser treatment in patients with bilateral large drusen: the complications of age-related macular degeneration prevention trial. Ophthalmology. 2006;113:1974-86.

26. Friberg TR, Brennen PM, Freeman WR, Musch DC, Group PS. Prophylactic treatment of age-related macular degeneration report number 2: 810-nanometer laser to eyes with drusen: bilaterally eligible patients. Ophthalmic Surg Lasers Imaging. 2009;40:530-8.

27. Friberg TR, Musch DC, Lim JI, Morse L, Freeman W, Sinclair S, et al. Prophylactic treatment of age-related macular degeneration report number 1: 810-nanometer laser to eyes with drusen. Unilaterally eligible patients. Ophthalmology. 2006;113:622e621.

28. Olk RJ, Friberg TR, Stickney KL, Akduman L, Wong KL, Chen $\mathrm{MC}$, et al. Therapeutic benefits of infrared $(810-\mathrm{nm})$ diode laser macular grid photocoagulation in prophylactic treatment of nonexudative age-related macular degeneration: two-year results of a randomized pilot study. Ophthalmology. 1999;106:2082-90.

29. Frennesson CI, Bek T, Jaakkola A, Nilsson SE, Prophylactic Laser Treatment Study Group. Prophylactic laser treatment of soft drusen maculopathy: a prospective, randomized Nordic study. Acta Ophthalmol. 2009;87:720-4.

30. Owens SL, Guymer RH, Gross-Jendroska M, Bird AC. Fluorescein angiographic abnormalities after prophylactic macular photocoagulation for high-risk age-related maculopathy. Am J Ophthalmol. 1999;127:681-7.
31. Parodi MB, Virgili G, Evans JR. Laser treatment of drusen to prevent progression to advanced age-related macular degeneration. Cochrane Database Syst Rev. 2009;3:CD006537.

32. Virgili G, Michelessi M, Parodi MB, Bacherini D, Evans JR. Laser treatment of drusen to prevent progression to advanced agerelated macular degeneration. Cochrane Database Syst Rev. 2015;10:CD006537

33. Mojana F, Brar M, Cheng L, Bartsch DU, Freeman WR. Longterm SD-OCT/SLO imaging of neuroretina and retinal pigment epithelium after subthreshold infrared laser treatment of drusen. Retina. 2011;31:235-42.

34. Roider J, Hillenkamp F, Flotte T, Birngruber R. Microphotocoagulation: selective effects of repetitive short laser pulses. Proc Natl Acad Sci USA. 1993;90:8643-7.

35. Roider J, Michaud NA, Flotte TJ, Birngruber R. Response of the retinal pigment epithelium to selective photocoagulation. Arch Ophthalmol. 1992;110:1786-92.

36. Brinkmann R, Huttmann G, Rogener J, Roider J, Birngruber R, Lin CP. Origin of retinal pigment epithelium cell damage by pulsed laser irradiance in the nanosecond to microsecond time regimen. Lasers Surg Med. 2000;27:451-64.

37. Kim HD, Jang SY, Lee SH, Kim YS, Ohn YH, Brinkmann R. et al.Retinal pigment epithelium responses to selective retina therapy in mouse eyes.Invest Ophthalmol Vis Sci. 2016;57:3486-95.

38. Kim HD, Han JW, Ohn YH, Brinkmann R, Park TK. Functional evaluation using multifocal electroretinogram after selective retina therapy with a microsecond-pulsed laser. Invest Ophthalmol Vis Sci. 2014;56:122-31.

39. Roider J, Brinkmann R, Wirbelauer C, Laqua H, Birngruber R. Retinal sparing by selective retinal pigment epithelial photocoagulation. Arch Ophthalmol. 1999;117:1028-34.

40. Park YG, Kim JR, Kang S, Seifert E, Theisen-Kunde D, Brinkmann R, et al. Safety and efficacy of selective retina therapy (SRT) for the treatment of diabetic macular edema in Korean patients. Graefes Arch Clin Exp Ophthalmol. 2016;254:1703-13.

41. Brinkmann R, Roider J, Birngruber R Selective retina therapy (SRT): a review on methods, techniques, preclinical and first clinical results. Bull Soc Belge Ophtalmol 2006;302:51-69.

42. Luttrull JK, Sramek C, Palanker D, Spink CJ, Musch DC. Longterm safety, high-resolution imaging, and tissue temperature modeling of subvisible diode micropulse photocoagulation for retinovascular macular edema. Retina. 2012;32:375-86.

43. Luttrull JK, Margolis BW. Functionally guided retinal protective therapy for dry age-related macular and inherited retinal degenerations: a pilot study. Invest Ophthalmol Vis Sci. 2016;57:265-75.

44. Johnson TM, Glaser BM. Micropulse laser treatment of retinalchoroidal anastomoses in age-related macular degeneration. Graefes Arch Clin Exp Ophthalmol. 2005;243:570-5.

45. Luttrull JK, Chang DB, Margolis BW, Dorin G, Luttrull DK. Laser resensitization of medically unresponsive neovascular agerelated macular degeneration: efficacy and implications. Retina. 2015;35:1184-94.

46. Wood JP, Plunkett M, Previn V, Chidlow G, Casson RJ. Nanosecond pulse lasers for retinal applications. Lasers Surg Med. 2011;43:499-510.

47. Guymer RH, Brassington KH, Dimitrov P, Makeyeva G, Plunkett $\mathrm{M}, \mathrm{Xia} \mathrm{W}$, et al. Nanosecond-laser application in intermediate AMD: 12-month results of fundus appearance and macular function. Clin Exp Ophthalmol. 2014;42:466-79.

48. Jobling AI, Guymer RH, Vessey KA, Greferath U, Mills SA, Brassington $\mathrm{KH}$, et al. Nanosecond laser therapy reverses pathologic and molecular changes in age-related macular degeneration without retinal damage. FASEB J. 2015;29:696-710. 
49. Klein ML, Ferris FL 3rd, Armstrong J, Hwang TS, Chew EY, Bressler SB, et al. Retinal precursors and the development of geographic atrophy in age-related macular degeneration. Ophthalmology. 2008;115:1026-31.

50. Toy BC, Krishnadev N, Indaram M, Cunningham D, Cukras CA, Chew EY, et al. Drusen regression is associated with local changes in fundus autofluorescence in intermediate age-related macular degeneration. Am J Ophthalmol. 2013;156:532-42 e531.

51. Chidlow G, Shibeeb O, Plunkett M, Casson RJ, Wood JP. Glial cell and inflammatory responses to retinal laser treatment: comparison of a conventional photocoagulator and a novel, 3nanosecond pulse laser. Invest Ophthalmol Vis Sci. 2013;54: 2319-32.
52. Wood JP, Shibeeb O, Plunkett M, Casson RJ, Chidlow G. Retinal damage profiles and neuronal effects of laser treatment: comparison of a conventional photocoagulator and a novel 3-nanosecond pulse laser. Invest Ophthalmol Vis Sci. 2013;54:2305-18.

53. Zhang JJ, Sun Y, Hussain AA, Marshall J. Laser-mediated activation of human retinal pigment epithelial cells and concomitant release of matrix metalloproteinases. Invest Ophthalmol Vis Sci. 2012;53:2928-37.

54. Lek JJ, Brassington KH, Luu CD, Chen FK, Arnold JJ, Heriot WJ, et al. Subthreshold nanosecond laser intervention in intermediate age-related macular degeneration. Ophthalmol Retin. 2017;1: 227-39. 